\title{
Endoscopic approach for the treatment of bariatric surgery complications
}

\author{
Jessica Ardila-Gatas, Aurora Pryor \\ Division of Bariatric, Foregut and Advanced Gastrointestinal Surgery, Department of Surgery, Stony Brook University Medical \\ Center, Stony Brook, New York, NY 11794-8191, USA. \\ Correspondence to: Dr. Jessica Ardila-Gatas, Division of Bariatric, Foregut and Advanced Gastrointestinal Surgery, Department \\ of Surgery, Stony Brook University Medical Center, Stony Brook, New York, NY 11794-8191, USA. \\ E-mail: jessica.ardila-gatas@stonybrookmedicine.edu
}

How to cite this article: Ardila-Gatas J, Pryor A. Endoscopic approach for the treatment of bariatric surgery complications. Mini-invasive Surg 2020;4:16. http://dx.doi.org/10.20517/2574-1225.2019.69

Received: 27 Dec 2019 First Decision: 3 Feb 2020 Revised: 4 Feb 2020 Accepted: 7 Feb 2020 Published: 11 Mar 2020

Science Editor: Wah Yang Copy Editor: Jing-Wen Zhang Production Editor: Tian Zhang

\begin{abstract}
The incidence of bariatric surgery is increasing exponentially. The number of bariatric surgeries performed in the United States has significantly increased in the past decades. Complications of bariatric surgery can present days to years postoperatively. Advances in endoscopic procedures and technology has made it possible to address many complications endoscopically. We describe the most common complications after bariatric surgery and the endoscopic treatment options available to date.
\end{abstract}

Keywords: Bariatric surgery, advanced endoscopy, intraluminal surgery

\section{INTRODUCTION}

Obesity is a public health problem ${ }^{[1]}$. The number of bariatric procedures performed in the United States has increased significantly in the past decades ${ }^{[1,2]}$. Laparoscopic Roux-en-Y gastric bypass (RYGB) and laparoscopic sleeve gastrectomy (LSG) are the most common bariatric procedures performed ${ }^{[2,3]}$. The overall mortality rate of bariatric surgery is $<0.2 \%$, yet the morbidity rate is between $4 \%$ and $10 \%$ with complications presenting most commonly within the first 30 days after surgery ${ }^{[2-4]}$. Some of the postoperative complications may be managed intraluminally using advances in surgical and interventional endoscopy $^{[2-6]}$.

Complications can be divided into early $\left(<30\right.$ days) or late $(>30 \text { days })^{[3,7]}$. Some can be encountered after any bariatric procedure and others are procedure specific ${ }^{[3]}$. The cornerstone for the diagnosis of luminal

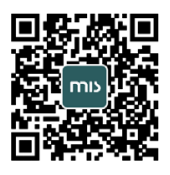


complications after weight loss surgery is esophagogastroduodenoscopy (EGD). Diagnostic and therapeutic EGD should not be delayed for fear of disruption of a fresh anastomosis. Evidence has shown it is safe and cost-effective to perform upper endoscopy in the early postoperative period ${ }^{[8]}$.

\section{BLEEDING}

Acute or early gastrointestinal (GI) hemorrhage usually presents within the first hours to days after surgery and it is often secondary to technical error ${ }^{[2,3]}$. Its incidence is $1 \%-4 \%^{[9]}$. Although bleeding usually occurs from the submucosal vessels along the staple line at the gastrojejunostomy, jejunojejunostomy, or the sleeve staple line, it can occur anywhere along the GI tract. Possible sites of bleeding include the gastric pouch and the gastric remnant, as well as extraluminal, at trocar insertion sites, dissection planes, or mesenteric or omental transection areas ${ }^{[2-4]}$. Late bleeding is usually caused by marginal ulceration or erosion (discussed below) $^{[5,9]}$.

Signs and symptoms of early postoperative bleeding include tachycardia, hemoglobin level drop, hematemesis, or hematochezia ${ }^{[9]}$. Hemodynamically stable patients are initially treated non-operatively with fluid resuscitation, close monitoring, proton pump inhibitors (PPIs), and blood transfusion as needed $^{[2-4,6,9]}$. For patients who present hemodynamically unstable, further operative or endoscopic procedures are warranted $^{[2-4]}$.

Different endoscopic treatments are available to manage a bleed: injection of diluted epinephrine or sclerosing agents, application of hemoclips or larger bear claw clips (Over-the-scope-clip, OTSC, Ovesco), thermal therapies (heater probe, mono- and bipolar electrocoagulation, argon plasma coagulation, and laser therapy), and application of hemostatic powder, fibrin, or thrombin glues ${ }^{[2-6]}$. Standard endoscopes can reach proximal bleeders in the gastric pouch or the sleeve staple line. For distal bleeders, balloon- or spiral-assisted enteroscopy, or even surgical assistance, may be needed to reach the jejunojejunostomy or the gastric remnant ${ }^{[2,3]}$.

\section{LEAKS AND FISTULAS}

Leaks commonly occur at the anastomosis or staple line ${ }^{[2]}$. After RYGB, leaks are usually seen at the gastrojejunal (GJ) anastomosis, in up to $2 \%-5 \%$ of cases $^{[2,5]}$ but can occur at any staple line or other location on the GI tract. After LSG, leaks are most common near the angle of His, where the staple line meets the gastroesophageal junction ${ }^{[2,3]}$. This is attributed to distal stenosis, increased proximal pressure, thinner tissue, and relative vascular watershed on angiographic studies, and occurs in $1 \%-9 \%$ of $\operatorname{cases}^{[2,4]}$. After duodenal switch, leaks may also be seen at the duodenojejunal (DJ) anastomosis.

Leaks are associated with significant morbidity and mortality. Although rare, with an incidence of $1 \%-6 \%$, several factors are believed to contribute in their development ${ }^{[2-4]}$. Ischemia, technical error such as overlapping staple lines, or anastomotic tension are suspected among the factors that leads to leaks ${ }^{[2]}$. Fistulas are defined as an abnormal communication between the GI tract and another organ (in the abdomen or thorax) or surface $e^{[7,10]}$. Generally, fistulas are related to acute leaks that fail to close in more than 12 weeks ${ }^{[7]}$. Complications after RYGB are gastrogastric fistulas between the gastric pouch and remnant, fistulas to the surrounding viscous organs, or fistulas to the $\operatorname{skin}^{[10]}$.

Signs and symptoms of leaks include abdominal pain, fevers, and tachycardia ${ }^{[2]}$. Suspicion of a leak requires thorough work up to assess the location and size of the defect, infection control with antibiotics, nutritional optimization, and appropriate therapeutic intervention ${ }^{[2,4]}$. A CT scan is usually required to assess for intraabdominal fluid collections. If there is any surrounding fluid collection distant to the GI lumen, this needs to be drained by interventional radiology, laparoscopically, or transluminal endoscopic debridement and 


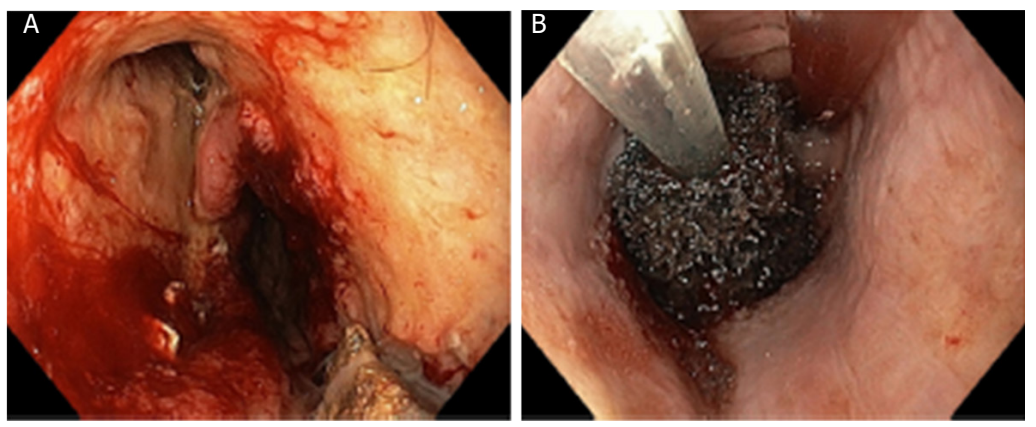

Figure 1. Leakage Endo-Sponge treatment ${ }^{[6]}$. A: evidence of fistula; B: placement of Endo-Sponge treatment

drainage (by endo-vacuum or with pigtail catheters) [Figure 1] ${ }^{[2-5,7]}$. Depending on the size of the fistula, different approaches can be taken. The key goals of endoscopic treatment are to cover (self-expandable metallic stents, SEMS) or close the fistula (de-epithelialization, clips, endoscopic suturing (Overstitch), and secondary intention with aid of a vacuum or septotomy) ${ }^{[7]}$. Small fistulas can be closed with OTSC ${ }^{[2,4,5]}$. Larger defects can be covered with stents or closed with sutures ${ }^{[2,4,5,7]}$, although surgical intervention may be required [Figure 2].

SEMS are the most commonly used endoscopic modality for leak treatment ${ }^{[2-6]}$. The self-expandable stents are placed over the leak area, isolating the area from the esophageal and gastric secretions, preventing further contamination and enhancing healing ${ }^{[2,6,7]}$. Patients can resume oral intake while the stent is in place, which enhances their nutrition and further healing. Stent placement is done under fluoroscopy and stents are later removed in 2-3 weeks to assess healing rate and prevent stent incorporation into the native tissue $^{[2-4]}$. Stent migration, described in $>40 \%$ of cases, is a possible complication with the usage of stents. Migration might require urgent endoscopy with stent removal and possible replacement. Modalities such as clips to minimize migration have been employed with some success. Endoscopic suturing, OTSCs, and glue injection have been used as adjuncts to stenting ${ }^{[2-4]}$. Systematic reviews and meta-analysis have been done to show the success of stenting, with a pooled proportion of successful leak closures of $87.77 \%{ }^{[11]}$.

\section{BEZOARS}

Bezoars consist of coagulated blood, undigestable fibers, undigested milk products, hair, or medications found intraluminally that do not pass through the GI tract ${ }^{[2]}$. Bezoars can be found following bariatric surgery and may lead to bowel obstruction. The incidence of bezoar-induced obstruction is unknown since the literature consists of mostly case reports. A stricture in the GJ anastomosis or foreign bodies at the staple line can serve as a nidus for bezoar formation. Endoscopy is used for diagnosis and treatment ${ }^{[2,4]}$. Techniques used to break the bezoar include water jet fragmentation, direct suction, and drills ${ }^{[2,5,6]}$.

\section{FAILURE TO THRIVE}

Placement of a nasogastric or nasojejunal feeding tubes can be done with endoscopy ${ }^{[6]}$. Patients who develop complications such as fistula or leak that need to be kept nil per os can maintain their calorie intake through enteral feeds. Placing the tube with endoscopic guidance prevents further tissue damage ${ }^{[4,6]}$.

\section{STRICTURE AND STENOSIS}

Stricture and stenosis peaks 3-4 weeks postoperatively and presents with dysphagia to solid food that progresses to intolerance to liquids ${ }^{[2,4]}$. Other symptoms include nausea, emesis, reflux, and epigastric pain $^{[7]}$. 

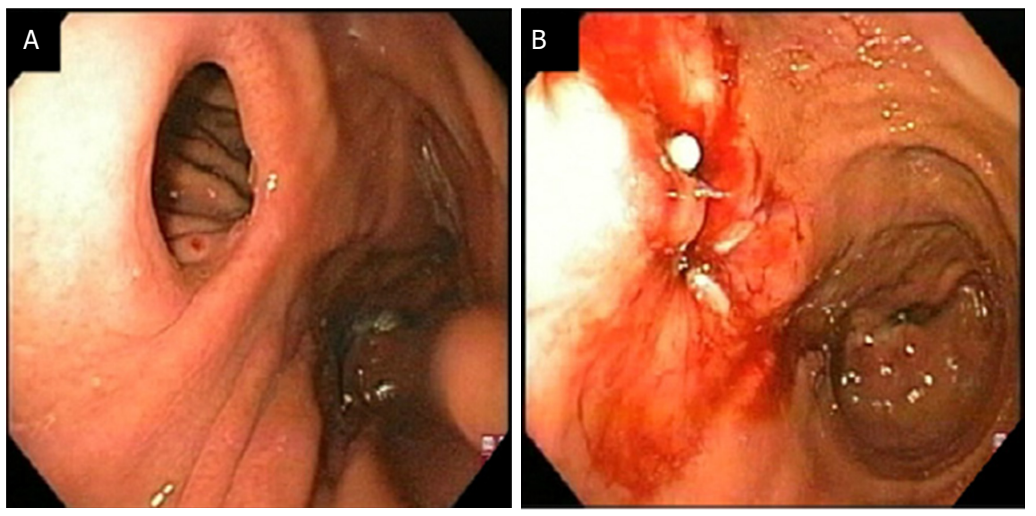

Figure 2. Gastrogastric fistula (B) after endoscopic repair ${ }^{[5]}$. A: evidence of gastrogastric fistula; B: after endoscopic repair

After RYGB, GJ anastomotic stricture is the most common site of primary strictures ${ }^{[7]}$. This is defined as a stoma that is $<10 \mathrm{~mm}$ in diameter. Stricture incidence is $3 \%-28 \%{ }^{[2,7]}$. Causes are multifactorial, including chemical agents [nonsteroidal anti-inflammatory drugs (NSAIDs) and tobacco], surgical technique (circular $v s$. linear stapler $v s$. hand sewn anastomosis), anastomotic tension and suture granuloma, among others ${ }^{[7]}$. The stricture can be classified by its endoscopic appearance into mild (allowing passage of a 10.5-mm endoscope), moderate (allowing passage of an $8.5-\mathrm{mm}$ pediatric endoscope), severe (allowing passage of a guidewire), or complete/near-complete obstruction (no passage of any instrumentation) ${ }^{[12]}$.

After LSG, stenosis can occur at the incisura angularis or gastroesophageal junction ${ }^{[7]}$. Sleeve stenosis occurs in between $0.1 \%$ and $3.9 \%$ of cases ${ }^{[2]}$. The causes are not clearly defined, but some reasons narrowing occurs are due to partial or complete over-sewing of the staple line or improper placement of the staple line (relative to the incisura or causing a torsion along its axis) ${ }^{[7]}$. Bougie size has not been found to be a factor contributing to strictures ${ }^{[7]}$.

Treatment consists of repetitive through the scope balloon dilation or bougienage in 10-14-day intervals ${ }^{[5,6]}$ [Figure 3]. One to two dilations to $18 \mathrm{~mm}$ are usually enough to achieve permanent patency of the anastomosis. If the stenosis is too narrow for the scope to pass, a guidewire is used for the balloon and bougie dilation under fluoroscopy ${ }^{[2,4,7]}$. These techniques give the endoscopist the ability to assess the resistance of the stenosis and decide if a larger balloon $v s$. bougie can be advanced. Strictures dilated within the first three months are more likely to be resolved with endoscopic dilation and less likely to require revisional surgery ${ }^{[7]}$. The GJ anastomotic size should not exceed $15 \mathrm{~mm}$; otherwise, the patient is at risk of weight regain ${ }^{[2-6]}$. Resistant strictures can be managed with endoscopic stricturoplasty and/or steroid injection. For Kenalog injection, $1 \mathrm{mg}$ of steroid is divided into four injections in the periphery of the stricture ${ }^{[13]}$.

A new endoscopic technique has been described for the treatment of strictures. A tunneled stricturotomy can be performed in experienced hands with good results in several case reports. Further studies are needed for long-term results ${ }^{[14]}$.

\section{MARGINAL ULCERS}

Ulceration is a late complication. Marginal ulcers are found on the jejunal side of the gastrojejunostomy in the RYGB patients ${ }^{[2]}$. Stomal ulcers are those that occur on the gastric side of the anastomosis and are believed to be caused by local ischemia. Marginal ulcer incidence is $2 \%-18 \%^{[2,4,15]}$. They are usually seen a few weeks or years after surgery. Risk factors for their development are poorly understood, but include poor blood supply to the anastomosis; presence of a foreign material (sutures or staples); use of NSAIDs, 

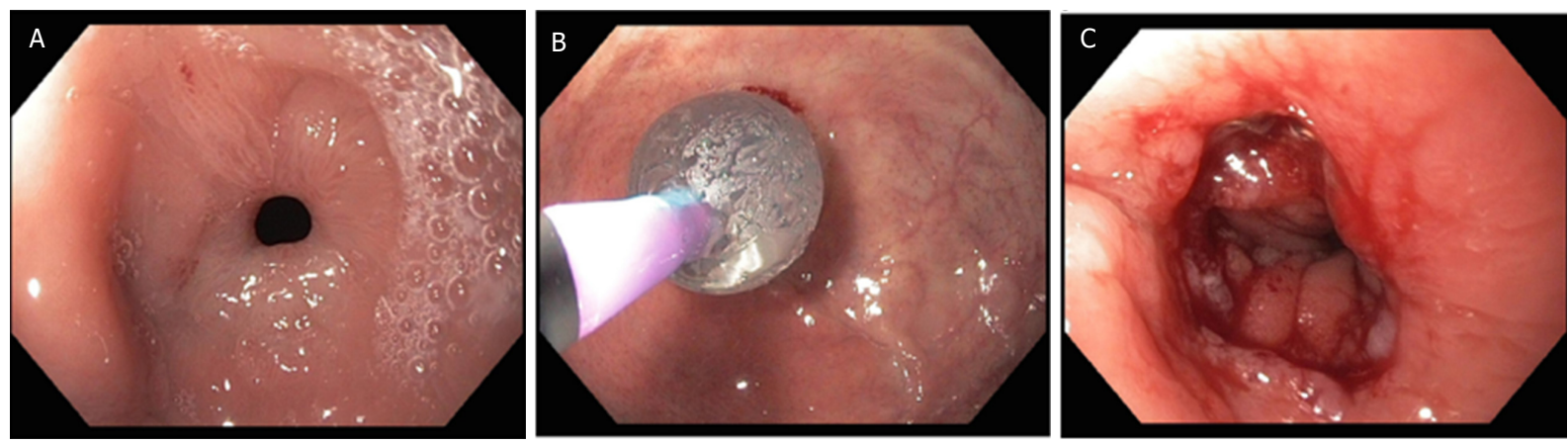

Figure 3. Anastomotic stenosis, before and after balloon dilation ${ }^{[6]}$. A: anastomotic stenosis; B: balloon dilation; C: anatomosis after dilation

steroids, tobacco, or alcohol; chemical inflammation due to gastric secretions; and Helicobacter pylori infection $^{[2-6]}$.

Prevention of marginal ulcers has been the focus of multiple bariatric publications. Avoiding NSAIDs, smoking cessation, and prophylactic PPIs have been the most widely used standard practices to reduce the incidence of marginal ulcers ${ }^{[2,3]}$. Treatment includes PPIs, sucralfate solution, and misoprostol (in patients who have been taking NSAIDs) ${ }^{[2,4,5,7]}$.

EGD has been used to aid in the diagnosis and to elucidate the etiology of the ulcers. If a foreign body is identified, it should be removed to facilitate healing ${ }^{[5]}$. These can be achieved by using over the scope grasping forceps, rat-tooth forceps, or standard endoscopic scissors ${ }^{[2]}$. Repeat EGD should be performed to confirm healing of marginal ulcers. Non-healing or recurrent ulcers should prompt investigation of underlying problems such as gastrogastric fistula as the cause of the ulcer ${ }^{[5,6]}$. Ulcers that persist despite medical therapy should be considered for surgical management.

\section{WEIGHT REGAIN}

Inadequate weight loss or failure to respond to bariatric surgery is multifactorial and must be addressed with a multidisciplinary approach. Different factors have been identified: medical (anatomic factors, nutritional deficiencies, and metabolic parameters), psychological (emotional ties to cravings and food addiction), or educational (dietitian counseling, preoperative weight loss goals, calorie counting, and noncompliance to follow up ${ }^{[7]}$.

A dilated GJ anastomosis has been associated with weight regain. This is often identified within the first two years after surgery ${ }^{[7]}$. Multiple endoscopic techniques have been described with limited success $s^{[2,7,16]}$.

Endoscopic narrowing of the anastomosis can be facilitated with a variety of techniques. Some techniques, such as injection of sclerosing agents, have been abandoned due to limited success or complications ${ }^{[2-4,16]}$. Using the OTSC or the Overstitch device are newer techniques that can be used over the scope to reduce the stoma size ${ }^{[1,1,17]}$. Long-term published outcomes from these techniques are limited ${ }^{[2-6,17]}$.

\section{BILIARY DISEASE}

Choledocholithiasis is frequently encountered in bariatric surgery patients, both preoperatively and postoperatively ${ }^{[2]}$. Common bile duct stones extraction after LSG is usually achievable using a standard approach; in contrast, getting access to the papilla in patients with RYGB anatomy is difficult ${ }^{[2-4]}$. In skillful 


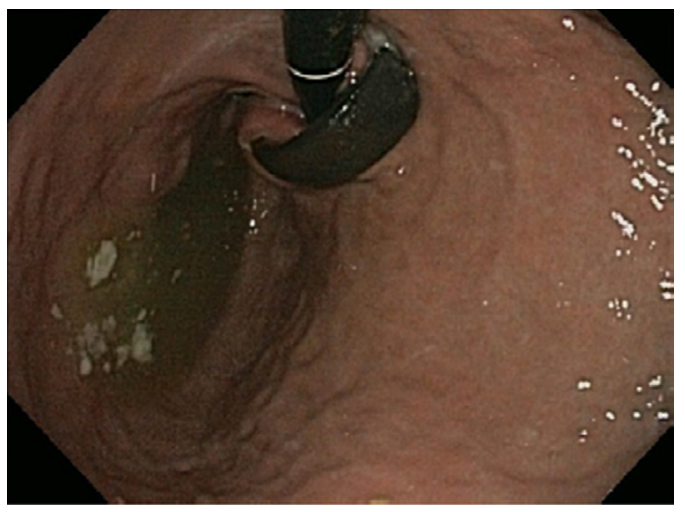

Figure 4. Band erosion ${ }^{[5]}$

endoscopist hands, an endoscopic retrograde cholangiopancreatography (ERCP) is successful $60 \%$ of the time in these patients ${ }^{[2-4]}$. The most common route used is to laparoscopically get access to the gastric remnant and through there get access to the papilla. An alternative is to use endoscopic ultrasound to create a gastrogastric fistula with SEMS placement, through which the scope can enable access to the papilla and subsequent $\mathrm{ERCP}^{[2,6]}$. Closure of the resultant gastrogastric fistula following this procedure is not well studied.

\section{BAND EROSION}

Even though laparoscopic gastric banding has decreased in popularity due to its long-term complications and lack of sustained weight loss, its complications are still relatively common presentations in bariatric centers.

Transmural migration of the band through the gastric wall occurs in $7 \%$ of gastric banding patients ${ }^{[2]}$. Endoscopy plays a role in the diagnosis and treatment of this complication. Endoscopic removal of eroded bands has been described ${ }^{[2-4]}$. With the use of ultrasonic shears, or preferably placing a wire around the band and using an ERCP rescue device, the band and tubing complex can be cut and removed transorally ${ }^{[18]}$ [Figure 4]. Endoscopic removal is most likely to be successful if the band buckle is within the gastric lumen. Traditionally, removal of the band is performed with a combination of laparoscopy and endoscopy $^{[2,18]}$.

\section{GASTROESOPHAGEAL REFLUX DISEASE}

As the rate of sleeve gastrectomy procedures performed in the US increases, the rate of de novo gastroesophageal reflux disease (GERD) after surgery and new-onset Barrett's esophagus has increased ${ }^{[4,5]}$. The use of novel endoscopic techniques to address GERD after bariatric surgery has slowly gained popularity. Several case reports have been published with successful results. The use of radiofrequency energy (Stretta) is the most widely described. The antireflux mucosectomy procedure involves endoscopic mucosal resection of the gastroesophagic junction and is also described ${ }^{[10,19]}$. The healing of the mucosal defect stimulates scar formation that improves reflux ${ }^{[19]}$. Further studies are needed to evaluate the longterm success of this approach.

\section{SUMMARY}

As the incidence of obesity increases exponentially, so does the incidence of bariatric surgery performed in the US. Complications of these procedures can present days to years postoperatively. Many of these complications can be managed endoscopically. Advances in endoscopic techniques have facilitated a 
minimally invasive approach with successful results. Upper endoscopy has been shown to be safe and cost effective in the diagnosis and treatment of bariatric surgery complications in the early and late postoperative period.

\section{DECLARATIONS}

\section{Authors' contributions}

Contributed to the design of the research, drafting of the manuscript and critical revision: Ardila-Gatas J, Pryor A

\section{Availability of data and materials}

Not applicable.

\section{Financial support and sponsorship}

None.

\section{Conflicts of interest}

All authors declared that there are no conflicts of interest.

\section{Ethical approval and consent to participate}

Not applicable.

\section{Consent for publication}

Not applicable.

\section{Copyright}

(c) The Author(s) 2020.

\section{REFERENCES}

1. Ward ZJ, Bleich SN, Cradock AL, Barrett JL, Giles CM, et al. Projected U.S. state-level prevalence of adult obesity and severe obesity. N Engl J Med 2019;381:2440-50.

2. Cai JX, Schweitzer MA, Kumbhari V. Endoscopic management of bariatric surgery complications. Surg Laparosc Endosc Percutan Tech 2016;26:93-101.

3. Boules M, Chang J, Haskins IN, Sharma G, Froylich D, et al. Endoscopic management of post-bariatric surgery complications. World J Gastrointest Endosc 2016;8:591-9.

4. da Rocha LCM, Pérez OAA, Arantes V. Endoscopic management of bariatric surgery complications: what the gastroenterologist should know. Rev Gastroenterol Mex 2016;81:35-47.

5. Kumar N, Thompson CC. Endoscopic management of complications after gastrointestinal weight loss surgery. Clin Gastroenterol Hepatol 2013;11:343-53.

6. Valli PV, Gubler C. Review article including treatment algorithm: endoscopic treatment of luminal complications after bariatric surgery. Clin Obes 2017;7:115-22.

7. ASMBS-SAGES. Bariatric endoscopy skill acquisition focused evaluation (BE-SAFE) 2019. Available from: https://besafe.asmbs.org/ [Last accessed on $14 \mathrm{Feb}$ 2020]

8. Sharma G, Ardila-Gatas J, Boules M, Davis M, Villamere J, et al. Upper gastrointestinal endoscopy is safe and feasible in the early postoperative period after Roux-en-Y gastric bypass. Surgery 2016;160:885-91.

9. Heneghan HM, Meron-Eldar S, Yenumula P, Rogula T, Brethauer SA, et al. Incidence and management of bleeding complications after gastric bypass surgery in the morbidly obese. Surg Obes Relat Dis 2012;8:729-35.

10. Fernandez-Esparrach G, Lautz DB, Thompson CC. Endoscopic repair of gastrogastric fistula after Roux-en-Y gastric bypass: a lessinvasive approach. Surg Obes Relat Dis 2010;6:282-8.

11. Puli SR, Spofford IS, Thompson CC. Use of self-expandable stents in the treatment of bariatric surgery leaks: a systematic review and meta-analysis. Gastrointest Endosc 2012;75:287-93.

12. Rosenthal RJ. Dilating the stenotic gastrojejunostomy after laparoscopic Roux-en-Y gastric bypass for morbid obesity: when things go wrong. J Gastrointest Surg 2009;13:1561-3.

13. Ramage Jr JI, Rumalla A, Baron TH, Pochron NL, Zinsmeister AR, et al. A prospective, randomized, double-blind, placebo-controlled trial of endoscopic steroid injection therapy for recalcitrant esophageal peptic strictures. Am J Gastroenterol 2005;100:2419-25.

14. de Moura DTH, Jirapinyo P, Aihara H, Thompson CC. Endoscopic tunneled stricturotomy in the treatment of stenosis after sleeve 
gastrectomy. VideoGIE 2019;4:68-71.

15. Spaniolas K, Yang J, Crowley S, Yin D, Docimo S, et al. Association of long-term anastomotic ulceration after Roux-en-Y gastric bypass with tobacco smoking. JAMA Surg 2018;153:862-4.

16. Dakin GF, Eid G, Mikami D, Pryor A, Chand B, et al. Endoluminal revision of gastric bypass for weight regain - a systematic review. Surg Obes Relat Dis 2013;9:335-42.

17. Thompson CC, Chand B, Chen YK, DeMarco DC, Miller L, et al. Endoscopic suturing for transoral outlet reduction increases weight loss after Roux-en-Y gastric bypass surgery. Gastroenterology 2013;145:129-37.e3.

18. El-Hayek K, Timratana P, Brethauer S, Chand B. Complete endoscopic/transgastric retrieval of eroded gastric band: description of a novel technique and review of the literature. Surg Endosc 2013;27:2974-9.

19. Hathorn KE, Jirapinyo P, Thompson CC. Endoscopic management of gastroesophageal reflux disease after sleeve gastrectomy by use of the antireflux mucosectomy procedure. VideoGIE 2019;4:251-3. 